\title{
Correction to: Advances in Mechanism and Machine Science
}

Tadeusz Uhl

\section{Correction to:}

T. Uhl (ed.), Advances in Mechanism and Machine Science, Mechanisms and Machine Science 73, https://doi.org/10.1007/978-3-030-20131-9

In the original version of the book, the following belated corrections have been incorporated:

In chapter 68, the author Samet Yavuz has been included.

In chapter 288, the author name "Hantao Liu" has been changed to "Haitao Liu".

In chapter 299, the author name "V. van der Tuleshov" has been changed to "V. van der Wijk". 